\title{
Probabilistic Sentence Reduction Using Support Vector Machines
}

\author{
Minh Le Nguyen, Akira Shimazu, Susumu Horiguchi \\ Bao Tu Ho and Masaru Fukushi
}

\author{
Japan Advanced Institute of Science and Technology \\ 1-8, Tatsunokuchi, Ishikawa, 923-1211, JAPAN \\ \{nguyenml, shimazu, hori, bao, mfukushi\}@jaist.ac.jp
}

\begin{abstract}
This paper investigates a novel application of support vector machines (SVMs) for sentence reduction. We also propose a new probabilistic sentence reduction method based on support vector machine learning. Experimental results show that the proposed methods outperform earlier methods in term of sentence reduction performance.
\end{abstract}

\section{Introduction}

The most popular methods of sentence reduction for text summarization are corpus based methods. Jing (Jing 00) developed a method to remove extraneous phrases from sentences by using multiple sources of knowledge to decide which phrases could be removed. However, while this method exploits a simple model for sentence reduction by using statistics computed from a corpus, a better model can be obtained by using a learning approach.

Knight and Marcu (Knight and Marcu 02) proposed a corpus based sentence reduction method using machine learning techniques. They discussed a noisy-channel based approach and a decision tree based approach to sentence reduction. Their algorithms provide the best way to scale up the full problem of sentence reduction using available data. However, these algorithms require that the word order of a given sentence and its reduced sentence are the same. Nguyen and Horiguchi (Nguyen and Horiguchi 03) presented a new sentence reduction technique based on a decision tree model without that constraint. They also indicated that semantic information is useful for sentence reduction tasks.

The major drawback of previous works on sentence reduction is that those methods are likely to output local optimal results, which may have lower accuracy. This problem is caused by the inherent sentence reduction model; that is, only a single reduced sentence can be obtained.
As pointed out by Lin (Lin 03), the best sentence reduction output for a single sentence is not approximately best for text summarization. This means that "local optimal" refers to the best reduced output for a single sentence, while the best reduced output for the whole text is "global optimal". Thus, it would be very valuable if the sentence reduction task could generate multiple reduced outputs and select the best one using the whole text document. However, such a sentence reduction method has not yet been proposed.

Support Vector Machines (Vapnik 95), on the other hand, are strong learning methods in comparison with decision tree learning and other learning methods (Sholkopf 97). The goal of this paper is to illustrate the potential of SVMs for enhancing the accuracy of sentence reduction in comparison with previous work. Accordingly, we describe a novel deterministic method for sentence reduction using SVMs and a twostage method using pairwise coupling (Hastie 98). To solve the problem of generating multiple best outputs, we propose a probabilistic sentence reduction model, in which a variant of probabilistic SVMs using a two-stage method with pairwise coupling is discussed.

The rest of this paper will be organized as follows: Section 2 introduces the Support Vector Machines learning. Section 3 describes the previous work on sentence reduction and our deterministic sentence reduction using SVMs. We also discuss remaining problems of deterministic sentence reduction. Section 4 presents a probabilistic sentence reduction method using support vector machines to solve this problem. Section 5 discusses implementation and our experimental results; Section 6 gives our conclusions and describes some problems that remain to be solved in the future. 


\section{Support Vector Machine}

Support vector machine (SVM)(Vapnik 95) is a technique of machine learning based on statistical learning theory. Suppose that we are given $l$ training examples $\left(x_{i}, y_{i}\right),(1 \leq i \leq l)$, where $x_{i}$ is a feature vector in $n$ dimensional feature space, $y_{i}$ is the class label $\{-1,+1\}$ of $x_{i}$. SVM finds a hyperplane $w \cdot x+b=0$ which correctly separates the training examples and has a maximum margin which is the distance between two hyperplanes $w \cdot x+b \geq 1$ and $w \cdot x+b \leq-1$. The optimal hyperplane with maximum margin can be obtained by solving the following quadratic programming.

$$
\begin{array}{lc}
\min & \frac{1}{2}\|w\|+C_{0} \sum_{i}^{l} \xi_{i} \\
\text { s.t. } & y_{i}\left(w \cdot x_{i}+b\right) \geq 1-\xi_{i} \\
\xi_{i} \geq 0 &
\end{array}
$$

where $C_{0}$ is the constant and $\xi_{i}$ is a slack variable for the non-separable case. In SVM, the optimal hyperplane is formulated as follows:

$$
f(x)=\operatorname{sign}\left(\sum_{1}^{l} \alpha_{i} y_{i} K\left(x_{i}, x\right)+b\right)
$$

where $\alpha_{i}$ is the Lagrange multiple, and $K\left(x^{\prime}, x^{\prime \prime}\right)$ is a kernel function, the SVM calculates similarity between two arguments $x^{\prime}$ and $x^{\prime \prime}$. For instance, the Polynomial kernel function is formulated as follow:

$$
K\left(x^{\prime}, x^{\prime \prime}\right)=\left(x^{\prime} \cdot x^{\prime \prime}\right)^{p}
$$

SVMs estimate the label of an unknown example $x$ whether the sign of $f(x)$ is positive or not.

\section{Deterministic Sentence Reduction Using SVMs}

\subsection{Problem Description}

In the corpus-based decision tree approach, a given input sentence is parsed into a syntax tree and the syntax tree is then transformed into a small tree to obtain a reduced sentence.

Let $t$ and $s$ be syntax trees of the original sentence and a reduced sentence, respectively. The process of transforming syntax tree $t$ to small tree $s$ is called "rewriting process" (Knight and Marcu 02), (Nguyen and Horiguchi 03). To transform the syntax tree $t$ to the syntax tree $s$, some terms and five rewriting actions are defined.

An Input list consists of a sequence of words subsumed by the tree $t$ where each word in the Input list is labelled with the name of all syntactic constituents in $t$. Let CSTACK be a stack that consists of sub trees in order to rewrite a small tree. Let RSTACK be a stack that consists of sub trees which are removed from the Input list in the rewriting process.

- SHIFT action transfers the first word from the Input list into CSTACK. It is written mathematically and given the label SHIFT.

- REDUCE $(l k, X)$ action pops the $l k$ syntactic trees located at the top of CSTACK and combines them in a new tree, where $l k$ is an integer and $X$ is a grammar symbol.

- DROP $X$ action moves subsequences of words that correspond to syntactic constituents from the Input list to RSTACK.

- ASSIGN TYPE $X$ action changes the label of trees at the top of the CSTACK. These POS tags might be different from the POS tags in the original sentence.

- RESTORE $X$ action takes the $X$ element in RSTACK and moves it into the Input list, where $X$ is a subtree.

For convenience, let configuration be a status of Input list, CSTACK and RSTACK. Let current context be the important information in a configuration. The important information are defined as a vector of features using heuristic methods as in (Knight and Marcu 02), (Nguyen and Horiguchi 03).

The main idea behind deterministic sentence reduction is that it uses a rule in the current context of the initial configuration to select a distinct action in order to rewrite an input sentence into a reduced sentence. After that, the current context is changed to a new context and the rewriting process is repeated for selecting an action that corresponds to the new context. The rewriting process is finished when it meets a termination condition. Here, one rule corresponds to the function that maps the current context to a rewriting action. These rules are learned automatically from the corpus of long sentences and their reduced sentences (Knight and Marcu 02), (Nguyen and Horiguchi 03).

\subsection{Example}

Figure 1 shows an example of applying a sequence of actions to rewrite the input sentence $(a, b, c, d, e)$, when each character is a word. It illustrates the structure of the Input list, two stacks, and the term of a rewriting process based on the actions mentioned above. For example, in the first row, DROP $\mathrm{H}$ deletes the sub-tree with its root node $\mathrm{H}$ in the Input list and stores 
it in the RSTACK. The reduced tree $s$ can be obtained after applying a sequence of actions as follows: DROP H; SHIFT; ASSIGN TYPE K; DROP B; SHIFT; ASSIGN TYPE H; REDUCE 2 F; RESTORE H; SHIFT; ASSIGN TYPE D; REDUCE $2 \mathrm{G}$. In this example, the reduced sentence is $(b, e, a)$.

\begin{tabular}{|c|c|c|c|c|c|}
\hline \multirow[t]{3}{*}{ RSTACK } & $\begin{array}{l}\text { INPUT LIST } \\
\text { ACTION }\end{array}$ & \multirow[t]{3}{*}{ Cstack } & \multirow{2}{*}{ 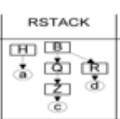 } & \multirow{2}{*}{$\begin{array}{l}\text { INPUT LST } \\
\text { ACTION } \\
\text { REDUCE } 2 F \\
\text { SHop } 7\end{array}$} & \multirow{2}{*}{$\frac{\text { CSTACK }}{\frac{[K]}{\dot{b}} \dot{\square}}$} \\
\hline & 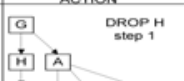 & & & & \\
\hline & 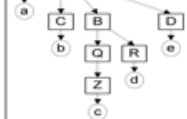 & & 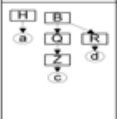 & $\begin{array}{l}\text { RESTORE H } \\
\text { Rtop }\end{array}$ & 界 \\
\hline \multirow[t]{3}{*}{ 田 } & \multirow{3}{*}{ 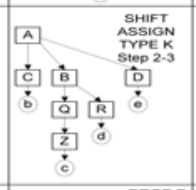 } & & \multirow{2}{*}{ 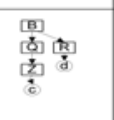 } & \multirow[t]{2}{*}{ 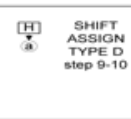 } & \multirow[t]{2}{*}{ 물 } \\
\hline & & & & & \\
\hline & & & \multirow{2}{*}{ 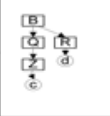 } & \multirow[t]{2}{*}{$\begin{array}{l}\text { REDUCEE } 20 \\
\text { Stop } 110\end{array}$} & \multirow{2}{*}{ 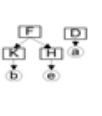 } \\
\hline \multirow{2}{*}{ 맘 } & \multirow{2}{*}{ 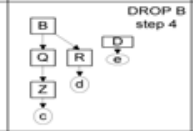 } & \multirow{2}{*}{$\frac{10}{6}$} & & & \\
\hline & & & \multirow{2}{*}{ 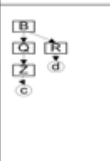 } & & \multirow{2}{*}{ smant troe } \\
\hline 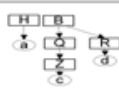 & 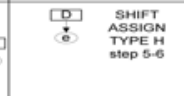 & \begin{tabular}{|l|}
$k$ \\
6 \\
6
\end{tabular} & & & \\
\hline
\end{tabular}

Figure 1: An Example of the Rewriting Process

\subsection{Learning Reduction Rules Using SVMs}

As mentioned above, the action for each configuration can be decided by using a learning rule, which maps a context to an action. To obtain such rules, the configuration is represented by a vector of features with a high dimension. After that, we estimate the training examples by using several support vector machines to deal with the multiple classification problem in sentence reduction.

\subsubsection{Features}

One important task in applying SVMs to text summarization is to define features. Here, we describe features used in our sentence reduction models.

The features are extracted based on the current context. As it can be seen in Figure 2, a context includes the status of the Input list and the status of CSTACK and RSTACK. We define a set of features for a current context as described bellow.

\section{Operation feature}

The set of features as described in (Nguyen and Horiguchi 03) are used in our sentence reduction models.

\section{Original tree features}

These features denote the syntactic constituents

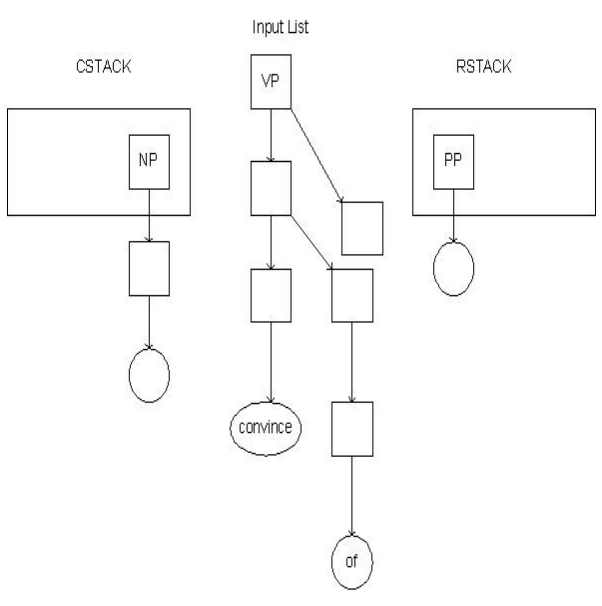

Figure 2: Example of Configuration

that start with the first unit in the Input list. For example, in Figure 2 the syntactic constituents are labels of the current element in the Input list from "VP" to the verb "convince".

\section{Semantic features}

The following features are used in our model as semantic information.

- Semantic information about current words within the Input list; these semantic types are obtained by using the named entities such as Location, Person, Organization and Time within the input sentence. To define these name entities, we use the method described in (Borthwick 99).

- Semantic information about whether or not the word in the Input list is a head word.

- Word relations, such as whether or not a word has a relationship with other words in the subcategorization table. These relations and the sub-categorization table are obtained using the Commlex database (Macleod 95).

Using the semantic information, we are able to avoid deleting important segments within the given input sentence. For instance, the main verb, the subject and the object are essential and for the noun phrase, the head noun is essential, but an adjective modifier of the head noun is not. For example, let us consider that the verb "convince" was extracted from the Comlex database as follows.

convince

NP-PP: PVAL ("of")

NP-TO-INF-OC

This entry indicates that the verb "convince" 
can be followed by a noun phrase and a prepositional phrase starting with the preposition "of". It can be also followed by a noun phrase and a to-infinite phrase. This information shows that we cannot delete an "of" prepositional phrase or a to-infinitive that is the part of the verb phrase.

\subsubsection{Two-stage SVM Learning using Pairwise Coupling}

Using these features we can extract training data for SVMs. Here, a sample in our training data consists of pairs of a feature vector and an action. The algorithm to extract training data from the training corpus is modified using the algorithm described in our pervious work (Nguyen and Horiguchi 03).

Since the original support vector machine (SVM) is a binary classification method, while the sentence reduction problem is formulated as multiple classification, we have to find a method to adapt support vector machines to this problem. For multi-class SVMs, one can use strategies such as one-vs all, pairwise comparison or $D A G$ graph (Hsu 02). In this paper, we use the pairwise strategy, which constructs a rule for discriminating pairs of classes and then selects the class with the most winning among two class decisions.

To boost the training time and the sentence reduction performance, we propose a two-stage SVM described below.

Suppose that the examples in training data are divided into five groups $m_{1}, m_{2}, \ldots, m_{5}$ according to their actions. Let Svmc be multiclass SVMs and let Svmc-i be multi-class SVMs for a group $m_{i}$. We use one Svmc classifier to identify the group to which a given context $e$ should be belong. Assume that $e$ belongs to the group $m_{i}$. The classifier Svmc-i is then used to recognize a specific action for the context $e$. The five classifiers Svmc-1, Svmc-2,..., Svmc-5 are trained by using those examples which have actions belonging to SHIFT, REDUCE, DROP, ASSIGN TYPE and RESTORE.

Table 1 shows the distribution of examples in five data groups.

\subsection{Disadvantage of Deterministic Sentence Reductions}

The idea of the deterministic algorithm is to use the rule for each current context to select the next action, and so on. The process terminates when a stop condition is met. If the early steps of this algorithm fail to select the best ac-
Table 1: Distribution of example data on five data groups

\begin{tabular}{|l|c|}
\hline Name & Number of examples \\
\hline SHIFT-GROUP & 13,363 \\
\hline REDUCE-GROUP & 11,406 \\
\hline DROP-GROUP & 4,216 \\
\hline ASSIGN-GROUP & 13,363 \\
\hline RESTORE-GROUP & 2,004 \\
\hline TOTAL & 44,352 \\
\hline
\end{tabular}

tions, then the possibility of obtaining a wrong reduced output becomes high.

One way to solve this problem is to select multiple actions that correspond to the context at each step in the rewriting process. However, the question that emerges here is how to determine which criteria to use in selecting multiple actions for a context. If this problem can be solved, then multiple best reduced outputs can be obtained for each input sentence and the best one will be selected by using the whole text document.

In the next section propose a model for selecting multiple actions for a context in sentence reduction as a probabilistic sentence reduction and present a variant of probabilistic sentence reduction.

\section{Probabilistic Sentence Reduction Using SVM}

\subsection{The Probabilistic SVM Models}

Let $A$ be a set of $k$ actions $A=$ $\left\{a_{1}, a_{2} \ldots a_{i}, \ldots, a_{k}\right\}$ and $C$ be a set of $n$ contexts $C=\left\{c_{1}, c_{2} \ldots c_{i}, \ldots, c_{n}\right\}$. A probabilistic model $\alpha$ for sentence reduction will select an action $a \in A$ for the context $c$ with probability $p^{\alpha}(a \mid c)$. The $p^{\alpha}(a \mid c)$ can be used to score action $a$ among possible actions $A$ depending the context $c$ that is available at the time of decision. There are several methods for estimating such scores; we have called these "probabilistic sentence reduction methods". The conditional probability $p^{\alpha}(a \mid c)$ is estimated using a variant of probabilistic support vector machine, which is described in the following sections.

\subsubsection{Probabilistic SVMs using Pairwise Coupling}

For convenience, we denote $u_{i j}=p\left(a=a_{i} \mid a=\right.$ $\left.a_{i} \vee a_{j}, c\right)$. Given a context $c$ and an action $a$, we assume that the estimated pairwise class probabilities $r_{i j}$ of $u_{i j}$ are available. Here $r_{i j}$ can be estimated by some binary classifiers. For instance, we could estimate $r_{i j}$ by using the 
SVM binary posterior probabilities as described in (Plat 2000). Then, the goal is to estimate $\left\{p_{i}\right\}_{i=1}^{k}$, where $p_{i}=p\left(a=a_{i} \mid c\right), i=1,2, \ldots, k$. For this propose, a simple estimate of these probabilities can be derived using the following voting method:

$$
p_{i}=2 \sum_{j: j \neq i} I_{\left\{r_{i j}>r_{j i}\right\}} / k(k-1)
$$

where $I$ is an indicator function and $k(k-1)$ is the number of pairwise classes. However, this model is too simple; we can obtain a better one with the following method.

Assume that $u_{i j}$ are pairwise probabilities of the model subject to the condition that $u_{i j}=$ $p_{i} /\left(p_{i}+p_{j}\right)$. In (Hastie 98), the authors proposed to minimize the Kullback-Leibler (KL) distance between the $r_{i j}$ and $u_{i j}$

$$
l(p)=\sum_{i \neq j} n_{i j} r_{i j} \log \frac{r_{i j}}{u_{i j}}
$$

where $r_{i j}$ and $u_{i j}$ are the probabilities of a pairwise $a_{i}$ and $a_{j}$ in the estimated model and in our model, respectively, and $n_{i j}$ is the number of training data in which their classes are $a_{i}$ or $a_{j}$. To find the minimizer of equation (6), they first calculate

$$
\frac{\partial l(p)}{\partial p_{i}}=\sum_{i \neq j} n_{i j}\left(-\frac{r_{i j}}{p_{i}}+\frac{1}{p_{i}+p_{j}}\right) .
$$

Thus, letting $\Delta l(p)=0$, they proposed to find a point satisfying

$$
\sum_{j: j \neq i} n_{i j} u_{i j}=\sum_{j: j \neq i} n_{i j} r_{i j}, \quad \sum_{i=1}^{k} p_{i}=1,
$$

where $i=1,2, \ldots k$ and $p_{i}>0$.

Such a point can be obtained by using an algorithm described elsewhere in (Hastie 98). We applied it to obtain a probabilistic SVM model for sentence reduction using a simple method as follows. Assume that our class labels belong to $l$ groups: $M=\left\{m_{1}, m_{2} \ldots m_{i}, \ldots, m_{l}\right\}$, where $l$ is a number of groups and $m_{i}$ is a group e.g., SHIFT, REDUCE ,..., ASSIGN TYPE. Then the probability $p(a \mid c)$ of an action $a$ for a given context $c$ can be estimated as follows.

$$
p(a \mid c)=p\left(m_{i} \mid c\right) \times p\left(a \mid c, m_{i}\right)
$$

where $m_{i}$ is a group and $a \in m_{i}$. Here, $p\left(m_{i} \mid c\right)$ and $p\left(a \mid c, m_{i}\right)$ are estimated by the method in (Hastie 98).

\subsection{Probabilistic sentence reduction algorithm}

After obtaining a probabilistic model $p$, we then use this model to define function score, by which the search procedure ranks the derivation of incomplete and complete reduced sentences. Let $d(s)=\left\{a_{1}, a_{2}, \ldots a_{d}\right\}$ be the derivation of a small tree $s$, where each action $a_{i}$ belongs to a set of possible actions. The score of $s$ is the product of the conditional probabilities of the individual actions in its derivation.

$$
\operatorname{Score}(s)=\prod_{a_{i} \in d(s)} p\left(a_{i} \mid c_{i}\right)
$$

where $c_{i}$ is the context in which $a_{i}$ was decided. The search heuristic tries to find the best reduced tree $s^{*}$ as follows:

$$
s^{*}=\underbrace{\operatorname{argmax}}_{s \in \operatorname{tree}(t)} \quad \text { Score }(s)
$$

where tree $(t)$ are all the complete reduced trees from the tree $t$ of the given long sentence. Assume that for each configuration the actions $\left\{a_{1}, a_{2}, \ldots a_{n}\right\}$ are sorted in decreasing order according to $p\left(a_{i} \mid c_{i}\right)$, in which $c_{i}$ is the context of that configuration. Algorithm 1 shows a probabilistic sentence reduction using the top K-BFS search algorithm. This algorithm uses a breadth-first search which does not expand the entire frontier, but instead expands at most the top $K$ scoring incomplete configurations in the frontier; it is terminated when it finds $M$ completed reduced sentences $(C L$ is a list of reduced trees), or when all hypotheses have been exhausted. A configuration is completed if and only if the Input list is empty and there is one tree in the CSTACK. Note that the function get-context $\left(h_{i}, j\right)$ obtains the current context of the $j^{\text {th }}$ configuration in $h_{i}$, where $h_{i}$ is a heap at step $i$. The function $\operatorname{Insert}(s, h)$ ensures that the heap $h$ is sorted according to the score of each element in $h$. Essentially, in implementation we can use a dictionary of contexts and actions observed from the training data in order to reduce the number of actions to explore for a current context.

\section{Experiments and Discussion}

We used the same corpus as described in (Knight and Marcu 02), which includes 1,067 pairs of sentences and their reductions. To evaluate sentence reduction algorithms, we randomly selected 32 pairs of sentences from our parallel corpus, which is refered to as the test corpus. The training corpus of 1,035 sentences extracted 44,352 examples, in which each training example corresponds to an action. The SVM tool, LibSVM (Chang 01) is applied to train our model. The training examples were 


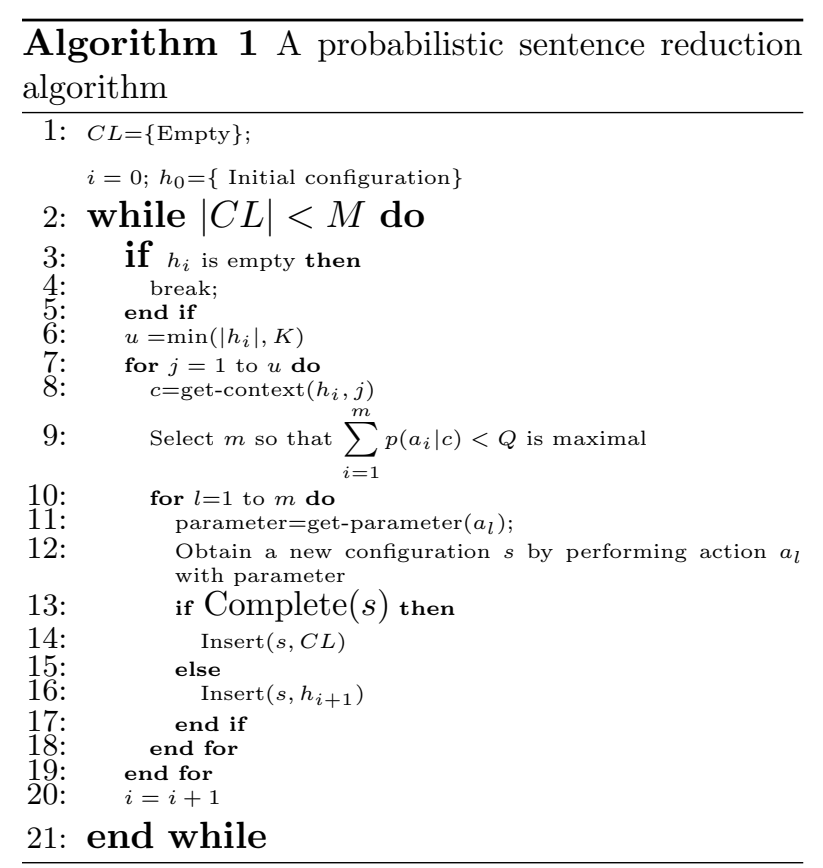

divided into SHIFT, REDUCE, DROP, RESTORE, and ASSIGN groups. To train our support vector model in each group, we used the pairwise method with the polynomial kernel function, in which the parameter $p$ in (3) and the constant $C_{0}$ in equation (1) are 2 and 0.0001 , respectively.

The algorithms (Knight and Marcu 02) and (Nguyen and Horiguchi 03) served as the baseline1 and the baseline2 for comparison with the proposed algorithms. Deterministic sentence reduction using SVM and probabilistic sentence reduction were named as SVM-D and SVMP, respectively. For convenience, the ten top reduced outputs using SVMP were called SVMP-10. We used the same evaluation method as described in (Knight and Marcu 02) to compare the proposed methods with previous methods. For this experiment, we presented each original sentence in the test corpus to three judges who are specialists in English, together with three sentence reductions: the human generated reduction sentence, the outputs of the proposed algorithms, and the output of the baseline algorithms.

The judges were told that all outputs were generated automatically. The order of the outputs was scrambled randomly across test cases. The judges participated in two experiments. In the first, they were asked to determine on a scale from 1 to 10 how well the systems did with respect to selecting the most important words in the original sentence. In the second, they were asked to determine the grammatical criteria of reduced sentences.

Table 2 shows the results of English language sentence reduction using a support vector machine compared with the baseline methods and with human reduction. Table 2 shows compression rates, and mean and standard deviation results across all judges, for each algorithm. The results show that the length of the reduced sentences using decision trees is shorter than using SVMs, and indicate that our new methods outperform the baseline algorithms in grammatical and importance criteria. Table 2 shows that the

Table 2: Experiment results with Test Corpus

\begin{tabular}{|l|c|c|c|}
\hline Method & Comp & Gramma & Impo \\
\hline Baseline1 & $57.19 \%$ & $8.60 \pm 2.8$ & $7.18 \pm 1.92$ \\
\hline Baseline2 & $57.15 \%$ & $8.60 \pm 2.1$ & $7.42 \pm 1.90$ \\
\hline SVM-D & $57.65 \%$ & $8.76 \pm 1.2$ & $7.53 \pm 1.53$ \\
\hline SVMP-10 & $57.51 \%$ & $8.80 \pm 1.3$ & $7.74 \pm 1.39$ \\
\hline Human & $64.00 \%$ & $9.05 \pm 0.3$ & $8.50 \pm 0.80$ \\
\hline
\end{tabular}

first 10 reduced sentences produced by SVMP10 (the SVM probabilistic model) obtained the highest performances. We also compared the computation time of sentence reduction using support vector machine with that in previous works. Table 3 shows that the computational times for SVM-D and SVMP-10 are slower than baseline, but it is acceptable for SVM-D.

Table 3: Computational times of performing reductions on test-set. Average sentence length was 21 words.

\begin{tabular}{|l|c|}
\hline Method & Computational times (sec) \\
\hline Baseline1 & 138.25 \\
\hline SVM-D & 212.46 \\
\hline SVMP-10 & 1030.25 \\
\hline
\end{tabular}

We also investigated how sensitive the proposed algorithms are with respect to the training data by carrying out the same experiment on sentences of different genres. We created the test corpus by selecting sentences from the web-site of the Benton Foundation (http://www.benton.org). The leading sentences in each news article were selected as the most relevant sentences to the summary of the news. We obtained 32 leading long sentences and 32 headlines for each item. The 32 sentences are used as a second test for our methods. We use a simple ranking criterion: the more the words in the reduced sentence overlap with the words in the headline, the more important the 
sentence is. A sentence satisfying this criterion is called a relevant candidate.

For a given sentence, we used a simple method, namely SVMP-R to obtain a reduced sentence by selecting a relevant candidate among the ten top reduced outputs using SVMP-10.

Table 4 depicts the experiment results for the baseline methods, SVM-D, SVMP-R, and SVMP-10. The results shows that, when applied to sentence of a different genre, the performance of SVMP-10 degrades smoothly, while the performance of the deterministic sentence reductions (the baselines and SVM deterministic) drops sharply. This indicates that the probabilistic sentence reduction using support vector machine is more stable.

Table 4 shows that the performance of SVMP-10 is also close to the human reduction outputs and is better than previous works. In addition, SVMP-R outperforms the deterministic sentence reduction algorithms and the differences between SVMP-R's results and SVMP10 are small. This indicates that we can obtain reduced sentences which are relevant to the headline, while ensuring the grammatical and the importance criteria compared to the original sentences.

Table 4: Experiment results with Benton Corpus

\begin{tabular}{|l|c|c|c|}
\hline Method & Comp & Gramma & Impo \\
\hline Baseline1 & $54.14 \%$ & $7.61 \pm 2.10$ & $6.74 \pm 1.92$ \\
\hline Baseline2 & $53.13 \%$ & $7.72 \pm 1.60$ & $7.02 \pm 1.90$ \\
\hline SVM-D & $56.64 \%$ & $7.86 \pm 1.20$ & $7.23 \pm 1.53$ \\
\hline SVMP-R & $58.31 \%$ & $8.25 \pm 1.30$ & $7.54 \pm 1.39$ \\
\hline SVMP-10 & $57.62 \%$ & $8.60 \pm 1.32$ & $7.71 \pm 1.41$ \\
\hline Human & $64.00 \%$ & $9.01 \pm 0.25$ & $8.40 \pm 0.60$ \\
\hline
\end{tabular}

\section{Conclusions}

We have presented a new probabilistic sentence reduction approach that enables a long sentence to be rewritten into reduced sentences based on support vector models. Our methods achieves better performance when compared with earlier methods. The proposed reduction approach can generate multiple best outputs. Experimental results showed that the top 10 reduced sentences returned by the reduction process might yield accuracies higher than previous work. We believe that a good ranking method might improve the sentence reduction performance further in a text.

\section{References}

A. Borthwick, "A Maximum Entropy Approach to Named Entity Recognition", Ph.D thesis, Computer Science Department, New York University (1999).

C.-C. Chang and C.-J. Lin, "LIBSVM: a library for support vector machines", Software available at http://www.csie.ntu.edu.tw/ cjlin/libsvm.

H. Jing, "Sentence reduction for automatic text summarization", In Proceedings of the First Annual Meeting of the North American Chapter of the Association for Computational Linguistics NAACL-2000.

T.T. Hastie and R. Tibshirani, "Classification by pairwise coupling", The Annals of Statistics, 26(1): pp. 451-471, 1998.

C.-W. Hsu and C.-J. Lin, "A comparison of methods for multi-class support vector machines", IEEE Transactions on Neural Networks, 13, pp. 415-425, 2002.

K. Knight and D. Marcu, "Summarization beyond sentence extraction: A Probabilistic approach to sentence compression", Artificial Intelligence 139: pp. 91-107, 2002.

C.Y. Lin, "Improving Summarization Performance by Sentence Compression - A Pilot Study", Proceedings of the Sixth International Workshop on Information Retrieval with Asian Languages, pp.1-8, 2003.

C. Macleod and R. Grishman, "COMMLEX syntax Reference Manual"; Proteus Project, New York University (1995).

M.L. Nguyen and S. Horiguchi, "A new sentence reduction based on Decision tree model", Proceedings of 17th Pacific Asia Conference on Language, Information and Computation, pp. 290-297, 2003

V. Vapnik, "The Natural of Statistical Learning Theory", New York: Springer-Verlag, 1995.

J. Platt, "Probabilistic outputs for support vector machines and comparison to regularized likelihood methods," in Advances in Large Margin Classifiers, Cambridege, MA: MIT Press, 2000.

B. Scholkopf et al, "Comparing Support Vector Machines with Gausian Kernels to Radius Basis Function Classifers", IEEE Trans. Signal Procesing, 45, pp. 2758-2765, 1997. 